\title{
Processing resources and age differences in working memory
}

\author{
ROBIN G. MORRIS, MARY L. GICK, and FERGUS I. M. CRAIK \\ University of Toronto, Toronto, Ontario, Canada
}

\begin{abstract}
This study investigated the performance of young and old subjects on a modified version of the working memory task developed by Baddeley and Hitch (1974). Subjects were required to verify a set of sentences of varying complexity while they repeated aloud zero, two, or four words. The older subjects took longer to verify the sentences, especially when the sentences were grammatically complex, but the effect of concurrent memory load on verification latency was the same in both groups. These results cast doubt on the notion that there is an age-related decline in one general pool of processing resources. They also suggest that older people have greater difficulty with the active processing aspects, rather than with the passive holding aspects, of working memory tasks.
\end{abstract}

In a review of research into age differences in human memory, Craik (1977) observed that deficits in short-term memory are negligible unless the memory task requires active manipulation of information or division of attention. In support of this position, Craik reviewed evidence showing that age differences are relatively slight in forward digit span, in free recall recency, and in the BrownPeterson paradigm, but that older people are clearly impaired on tasks such as backward digit span and dichotic listening. The more recently developed concept of working memory (Baddeley \& Hitch, 1974; Daneman \& Carpenter, 1980) typically refers to tasks in which subjects must divide their attention between ongoing processing and short-term storage. It should follow, therefore, that age decrements should be found in working memory tasks, and this result has been reported by several authors (Light \& Anderson, 1985; Spilich, 1983; Wright, 1981).

The purposes of the present study and of a parallel study reported by Gick, Craik, and Morris (1988) were to establish more precisely the locus of the age-related difficulty and to explore the factors contributing to the difficulty. With respect to the latter point, it is possible that older people perform poorly on working memory tasks because they are deficient in "processing resources" (Norman \& Bobrow, 1975). Craik and his colleagues (Craik, 1983; Craik \& Byrd, 1982) have suggested that older people have less "mental energy," or a smaller pool of processing resources on which to draw in order to execute required mental operations. According to this viewpoint, as the number of mental operations increases in a

This research was supported by a grant from the Natural Sciences and Engineering Research Council of Canada awarded to F. I. M. Craik. The authors would like to thank Lily Moysiuk and Lorna Morris for technical help. Mary Gick is now at the Department of Psychology, Carleton University, Ottawa, Canada. Requests for reprints should be sent to R. G. Morris, Department of Psychiatry, University of Newcastle upon Tyne, Royal Victoria Infirmary, Newcastle upon Tyne, NE1 4LP, England. given task, so does the competition for a limited resource pool. Older people are penalized because their processing resources are more readily exceeded, resulting in greater deficits on tasks that require complex processing or division of attention. This applies particularly to tests of working memory in which there is a necessary tradeoff between the processes associated with maintaining material in an active state and those required for carrying out other mental operations, such as a computational analysis of the material or executing a secondary task (Baddeley, 1986).

The notion that processing resources decline with age implies that any increase in complexity of the constituent operations should penalize older people to a greater extent. Gick et al. (1988) examined this issue, using the paradigm developed by Daneman and Carpenter (1980). Subjects were presented with a series of sentences; their task was to verify the accuracy of each sentence as it was presented, and also to recall the series of final words from each sentence after the entire series was presented. Task difficulty was varied in three ways: by contrasting paced and unpaced conditions, by varying the length of the sentence series, and by varying the grammatical complexity of the sentences used. It was predicted that older people would be penalized more than their younger counterparts by these manipulations. The results confirmed some, but not all, of these expectations, as will be described and discussed later in this article.

The present experiment used a modification of the "concurrent load" version of the working memory task developed by Baddeley and Hitch (1974). Subjects are first given a short list of unrelated words to hold in mind, and are then given a sentence to verify; finally, subjects recall the word list. The difficulty of the task may be varied both by increasing the number of words to be held in mind and by varying the grammatical complexity of the sentence to be verified. Baddeley and Hitch (1974) showed that both variables increased the time taken to verify the sen- 
tence. One problem with the paradigm is that subjects can switch their attention in unpredictable ways between the memory and verification operations. To counteract this tendency, we had subjects rehearse the word lists aloud in a continuous fashion while they verified the sentence. In this way, we hoped to reduce memory errors essentially to zero, and thus to make sentence verification latency and sentence verification errors the dependent measures of interest.

In the present study, young and old subjects were compared on a sentence verification task, similar to that used by Gick et al. (1988), with a concurrent memory load of zero, two, or four words. We predicted that two words would interfere minimally with sentence verification, since they could be maintained through articulatory rehearsal, which operates relatively automatically (Baddeley, Lewis, \& Vallar, 1984). We predicted that four words, however, would cause substantial interference, since four exceeds the number that can be held purely by articulatory rehearsal (Baddeley \& Hitch, 1974). If processing resources decrease with age, then substantial interference should be found in the older subjects in this condition. A further prediction is that the age-related working memory deficit should increase with increases in the grammatical complexity of the sentence verification task, since more complex tasks presumably make heavier demands on processing resources. This result was found by Wright (1981). In the present experiment, the complexity of the verification task was varied by making the sentence either active or passive and either positive or negative, following Baddeley and Hitch (1974).

\section{METHOD}

\section{Subjects}

Twenty-four younger and 24 older subjects served in the experiment. The younger subjects ( 21 females and 3 males) gained credit for participating as part of an introductory psychology course; their mean age was 19.7 years (range $=19-24$ years), mean years of education was 13.1 years $(S D=0.5$ years), and mean score on the Mill Hill Vocabulary Test was 13.5. The older subjects (10 females and 14 males) were taken from a pool of elderly volunteers living independently in the local community. Their mean age was 69.1 years (range $=60-80$ years), mean years of education was 13.1 years $(S D=3.5$ years), and mean Mill Hill score was 17.3. The vocabulary scores of the older subjects were significantly higher than those of the younger subjects $[t(47)=7.4, p<.01]$. When younger and older experimental participants are matched for years of education, it is typically found that the older group obtains higher scores on a vocabulary test.

\section{Material}

The word lists consisted of two or four high-frequency bisyllabic words (occurring at least 10 times in the corpus of the Kučera \& Francis, 1967, word norms). Eight types of sentence were presented, determined by whether the sentence was true or false, active or passive, and positive or negative, following the procedure used by Baddeley and Hitch (1974). The use of these manipulations to vary the grammatical complexity is typical of a wide range of reasoning tasks and is based on the assumption that the grammatical complexity of a sentence is related to the number of grammatical rules employed in its derivation (Wason \& Johnson-Laird, 1972). Although some controversy surrounds this issue (Fodor, Bever, \& Garrett, 1974), we have adopted the manipulations used by Baddeley and Hitch (1974) because they provide a useful heuristic for varying the difficulty of the sentences. The sentences were constructed so that their meanings would be readily accessible and contained material that was assumed to be widely known (e.g., "a sparrow can build a nest" or "a cat does not hunt mice"). The test items were taken from a total pool of 168 sentences, similar in nature to those used by Gick et al. (1988), but constructed independently.

\section{Design and Procedure}

In overview, younger and older subjects were given a single sentence to verify as rapidly as possible while simultaneously rehearsing zero, two, or four unrelated words. At the end of each trial, the subjects recalled the word list (when present) in the original serial order. The complexity of the sentence to be verified was varied as described above. Since the subjects rehearsed the memory list aloud throughout the trial, and since the maximum list length was only four words, we expected few recall errors; therefore, sentence verification latencies and errors formed the main dependent variables. Whereas sentence complexity was varied by having active or passive and positive or negative features, the data were collapsed over active/passive, and analyses are presented for positive versus negative sentences only. In line with previous work (Fodor et al., 1974), the positive/negative manipulation gave rise to larger differences in latency and errors than did the active/passive manipulation. Also, we have no theoretical interest in the nature of sentence complexity in the present context, since we wished only to vary the difficulty of the on-line processing task; collapsing the data over the active/passive manipulation simply clarifies the exposition. "Sentence complexity" thus refers to the positive/negative manipulation in the present study.

In greater detail, the experiment was designed with age as a between-subject factor and with two within-subject factors: concurrent memory load (zero, two, or four words) and sentence complexity (positive or negative sentence). The subjects were first given practice on the verification task, initially without a concurrent memory load, then with a two-word memory load, and finally with a four-word memory load. In each case, they were given eight trials, corresponding to the eight types of sentence (true/false $x$ active/passive $X$ positive/negative), arranged in random order. In the test phase of the experiment, the subjects were given nine sets of 16 sentences, with two of each sentence type in each set, arranged in random order for each subject. The nine sets were split into three series of three sets. In each series, there was one set with no concurrent memory load, one set with a two-word memory load, and one set with a four-word memory load, arranged in random order for each subject. Thus, for each memory preload condition, there were three sets of 16 sentences, one in each of the three series. The purpose of this design was to control for the effects of practice by spreading the trials of the different conditions across the experiment.

The material was presented visually on the monitor of a PET 8200 microcomputer (the computer also randomly allocated the sentences to the sets and ordered the sets according to the constraints given above). A 2-sec warning period signaled by the letter " $r$ " was first presented in the center of the screen. In the sets with the concurrent memory load, this was followed by the memory words, presented to the subject at a rate of one item per $2 \mathrm{sec}$. The subject was required to start cyclically repeating the items aloud and at a steady rate as soon as all of the words had been presented. Immediately after the last word was presented, the sentence appeared. The subject had a maximum of $8 \mathrm{sec}$ to respond by pressing one of two keys using his/her right and left index fingers, according to whether the sentence was true or false. Following the verification response, the sentence was replaced with a line of asterisks. The subject was required to keep rehearsing the memory words aloud in serial order 
for $4 \mathrm{sec}$ after the sentence verification response, at which point the asterisks were replaced by the word "stop." This procedure was used to ensure that the subjects continued rehearsing the memory words well past the sentence verification stage, and to enable the experimenter to ascertain more clearly what the subjects were articulating. In the control condition, without the concurrent load, the sentence immediately followed the warning period, and the trial was terminated by the subject's response.

\section{RESULTS}

\section{Memory Errors}

Performance was scored in terms of the percentage of words articulated in correct serial order by the subjects in the $4 \mathrm{sec}$ following sentence verification. In the condition with two memory words, errors were extremely infrequent (four in the whole experiment). The number of errors with four memory words was still relatively low and did not differ significantly across age groups or types of sentence (younger subjects: positive $=4.9 \%$, negative $=5.6 \%$; older subjects: positive $=4.6 \%$, negative $=4.3 \%$ ). A two-way analysis of variance (ANOVA) on these data showed that neither the effect of age $[F(1,46)$ $=0.38, M S e=5.99]$ nor the effect of sentence complexity $[F(2,138)=0.93, M S \mathrm{e}=0.92]$ was significant. The interaction between these two factors was also not significant $[F(2,138)=1.0, M S e=0.79]$.

\section{Verification Latencies}

Figure 1 shows verification latencies for correct responses in the two age groups. There is a tendency for the older subjects to have longer latencies, especially with the more complex negative sentences; also, increasing memory load is associated with longer latencies for both groups. The effects were assessed using a three-way ANOVA. This ANOVA showed main effects of age $[F(1,46)=6.35, p<.05, M S e=8.74]$, memory load $[F(2,92)=27.64, p<.001, M S e=2.18]$, and sentence complexity $[F(1,46)=104.67, p<.001, M S \mathrm{e}=0.31]$,

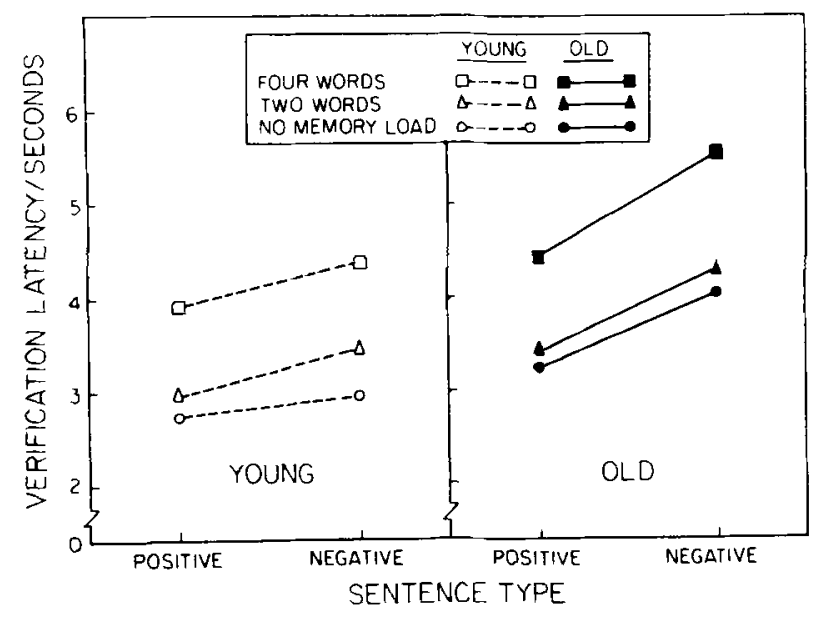

Figure 1. Sentence verification latencies for two age groups, for positive and negative sentences, and with varying memory loads.
Table 1

Error Rates (\%) for Young and Old Subjects on Sentence Verification with Varying Memory Loads

\begin{tabular}{|c|c|c|c|c|c|c|}
\hline & \multicolumn{2}{|c|}{ No Memory Load } & \multicolumn{2}{|c|}{2 Words } & \multicolumn{2}{|c|}{4 Words } \\
\hline & $\mathbf{P}$ & $\mathbf{N}$ & $\mathbf{P}$ & $\mathbf{N}$ & $\mathbf{P}$ & $\mathbf{N}$ \\
\hline \multicolumn{7}{|c|}{ Young Subjects } \\
\hline$M$ & 6.3 & 8.1 & 9.0 & 12.3 & 7.1 & 18.1 \\
\hline$S D$ & 6.7 & 8.7 & 9.6 & 11.1 & 8.6 & 12.7 \\
\hline \multicolumn{7}{|c|}{ Old Subjects } \\
\hline$M$ & 4.4 & 7.6 & 5.8 & 12.4 & 7.9 & 19.8 \\
\hline$S D$ & 5.4 & 7.7 & 7.3 & 9.7 & 9.1 & 17.2 \\
\hline
\end{tabular}

Note $-\mathrm{P}=$ positive sentences and $\mathrm{N}=$ negatjve sentences.

indicating that older subjects responded more slowly, and that increases in the memory load and in sentence complexity were associated with longer verification latencies. There was a significant interaction between memory load and sentence complexity $[F(2,92)=3.44, p<.05, M S \mathrm{e}$ $=0.14]$, showing that the effects of complexity were slightly larger with greater memory loads. There was no interaction between age and memory load, but age and sentence complexity interacted significantly $[F(1,46)=$ $20.90, p<.001, M S e=0.31$ ], indicating that the older subjects were comparatively slower when verifying the more complex sentences. Interestingly, then, verification latencies showed an interaction of age with one form of task difficulty (sentence complexity), but not with another (memory load).

\section{Verification Errors}

The percentages of sentence verification errors as a function of age and experimental condition are shown in Table 1 . These data were analyzed by means of a threeway ANOVA involving age, memory load, and sentence complexity. The analysis showed that there were no age differences in the number of errors $(F<1.0, M S e=$ 126.0); however, error rates increased both with memory load $[F(2,92)=17.7, p<.001, M S e=59.6]$ and with sentence complexity $[F(1,46)=37.6, p<.001, M S e=$ 75.2]. Memory load and sentence complexity interacted significantly $[F(2,92)=11.8, p<.001, M S e=43.3]$; the effects of complexity were again greater as memory load increased. Age interacted with neither sentence complexity nor memory load in this analysis.

\section{DISCUSSION}

The overall aim of the experiment was to explore the locus of the age-related decrement in working memory performance, and this was accomplished by means of a task combining sentence verification with concurrent articulation of short word lists in younger and older subjects. Since there were no age differences in the ability to rehearse overtly with two or four words, interest could be focused on sentence verification accuracy and latency. The major prediction was that if processing resources decline with age (Craik, 1983; Craik \& Byrd, 1982), then 
age differences in errors and verification latencies should be amplified both by increased sentence complexity and by increases in the concurrent memory load from zero to four words.

The results showed that the older subjects were slower overall to verify the sentences, although they were not less accurate. In line with prediction, verification latencies increased with an increase in sentence complexity, and this effect was stronger for the older group. The interaction of age and sentence complexity replicates the results of several previous studies, which have shown that increases in syntactic complexity are relatively more damaging to the comprehension processes of older adults (Emery, 1985; Kemper, 1987). The age $\times$ complexity interaction was not present in the verification error data, however.

In marked contrast to the effects of sentence complexity, increases in the concurrent memory load from zero to four words had no differential effect for older people on either latencies or errors. In agreement with Baddeley and Hitch (1974), both age groups showed a small increase in verification latency from no memory load to the twoword memory load, but a larger increase from the twoto the four-word memory load, consistent with the claim that two words can be rehearsed relatively automatically and thus do not interfere substantially with performance of a concurrent task (Baddeley et al., 1984). Although the results are more variable, a similar pattern is seen with errors, with an increase in the effect of more complex sentences at the higher memory load.

The parallel study by Gick et al. (1988) reported a very similar pattern of results. Gick et al. examined the performance of younger and older people on the Daneman and Carpenter (1980) working memory task, in which subjects verify a series of sentences and then recall the set of final words from each sentence. The memory scores are not comparable between the two studies, since in the present study every effort was made to keep such errors to a minimum. The subjects in the present study rehearsed the set of two or four words aloud, and made fewer than $5 \%$ recall errors overall; no effects of age or sentence complexity were found. The more demanding memory task used by Gick et al. resulted in significant effects of age, sentence complexity, and set size. In addition, the age $\times$ complexity interaction was significant, but the age $X$ set size interaction was not. When sentence verification latencies are considered, both studies found reliable effects of age, complexity, and set size; the age $\times$ set size interaction was not reliable in either study. The one difference in the latency data sets is that the age $\times$ complexity interaction was reliable in the present study, but was not in the Gick et al. experiment. However, in the sentence verification error data, the age $\times$ complexity interaction was significant in the Gick et al. study, but was not in the present study. Thus, older people had greater difficulty with the more complex sentences in both experiments, but this greater difficulty was revealed as more verification errors in the Gick et al. experiment and as increased verification latencies in the present study. Otherwise, the verification error data show similar patterns; both studies found reliable effects of sentence complexity and of set size, but no age $\times$ set size interaction. The one remaining difference in the error data sets is that the main effect of age was reliable in the Gick et al. study, but was not in the present experiment.

The interesting conclusion from both studies is that the deleterious effects of increased sentence complexity on performance are amplified in older people, but that the effects of increased memory load (set size in Gick et al., 1988) are not amplified by age. The absence of an age $x$ memory load interaction has two important implications for theories of cognitive aging. First, it sets limits on the notion that one general pool of processing resources declines with age. If it takes more resources to rehearse four words than two, and if older people are especially lacking in further resources, then the switch from two to four words should be more disruptive to concurrent performance for the older group; however, this result was not found. One possible reason for this null result is that many task-specific resource pools exist, rather than one general pool (Allport, 1980), and that aging affects those concerned with processing syntatic complexity, but not those concerned with maintaining words in working memory. A second possibility is that members of both age groups are already working at the limit of their capacity, and that the addition of extra items to the memory set has an equally deleterious effect on performance for all subjects. In any event, it seems clear that working memory performance cannot be considered to depend on a single set of processes; in this regard, the present results may help to dissect and document the various component processes (Baddeley, 1986).

The second implication of the present results for work on aging is that they also set limits on the claim that division of attention is necessarily more disruptive to the performance of older people (Craik, 1977). In both the present study and the Gick et al. (1988) experiment, no interactions were found between age and single-task/dualtask performance. In this respect, the results are in agreement with a recent study by Baddeley, Logie, Bressi, Della Sala, and Spinnler (1986), which shows that older people are not differentially impaired when memory span is combined with a tracking task adjusted to equate performance when the tasks are performed alone. On the other hand, the greater vulnerability of older people to interference in ongoing processing tasks that involve divided attention is well documented (McDowd \& Craik, in press; Salthouse, Rogan, \& Prill, 1984; Welford, 1958). At present, we can only speculate on the crucial difference between the two sets of studies; one possibility is that age-related decrements in division of attention do not appear when one task involves continuous rehearsal of a span or subspan list of verbal items.

In conclusion, it appears that the age-related decline in working memory performance found in the present experiment and in other studies (Gick et al., 1988; Light 
\& Anderson, 1985; Spilich, 1983; Wright, 1981) is not necessarily due to divided attention per se, as suggested originally by Craik (1977). Just as complexity was shown to have different effects in relation to age depending on the nature of the complexity in question, it seems possible that divided attention is also too broad a category to treat as a unitary factor. It seems, rather, that researchers must examine the component tasks and their characteristics before statements can be made about individual differences in divided attention performance.

In the context of short-term or working memory tasks, it seems possible that aging has relatively little effect on the ability to hold and rehearse verbal items, but that aging does have a deleterious effect on the ability to process further incoming information, especially when that material is complex. In the present study, increases in syntactic complexity were associated with differential agerelated increases in sentence verification latency. In the Gick et al. (1988) experiment, increases in complexity were associated with a differential age-related increase in verification errors and with a differential age-related decline in the ability to recall final words from the sentences. It seems, then, that older people have particular difficulty with the active processing aspects of working memory tasks, but are not differentially affected by increases in the difficulty of concurrent verbal rehearsal.

\section{REFERENCES}

AllPort, D. A. (1980). Patterns and actions: Cognitive mechanisms are content-specific. In G. Claxton (Ed.), Cognitive psychology: New directions (pp. 26-64). London: Routledge \& Kegan Paul.

BADDELEY, A. D. (1986). Working memory. London: Oxford University Press.

BadDeley, A. D., \& HiTCH, G. J. (1974). Working memory. In G. H. Bower (Ed.), The psychology of learning and motivation (Vol. 8, pp. 47-89). New York: Academic Press.

BadDeley, A. D., LeWIs, V., \& Vallar, B. (1984). Exploring the articulatory loop. Quarterly Journal of Experimental Psychology, 36, 233-252.

Baddeley, A. D., Logie, R., Bressi, S., Della Sala, S., \& SpinNLER, H. (1986). Dementia and working memory. The Quarterly Journal of Experimental Psychology, 38A, 603-618.
Craik, F. I. M. (1977). Age differences in human memory. In J. E. Birren \& K. W. Schaie (Eds.), Handbook of the psychology of aging (pp. 384-420). New York: Van Nostrand Reinhold.

CraIK, F. I. M. (1983). On the transfer of information from temporary to permanent memory. Philosophical Transactions of the Royal Society, London, 302B, 341-359.

CraIK, F. I. M., \& BYRD, M. (1982). Aging and cognitive deficits: The role of attentional resources. In F. I. M. Craik \& S. E. Trehub (Eds.), Aging and cognitive processes (pp. 191-211). New York: Plenum Press.

Daneman, M., \& Carpenter, P. (1980). Individual differences in working memory and reading. Journal of Verbal Learning \& Verbal Behavior, 19, 450-466.

EMERY, O. (1985). Language and aging. Experimental Aging Research, $11,3-60$.

Fodor, J. A., BeVer, T. G., \& GARRETT, M. F. (1974). The psychology of language. New York: McGraw-Hill.

Gick, M. L., Craik, F. I. M., \& MorRIs, R. G. (1988). Task complexity and age differences in working memory. Memory \& Cognition, 16, 353-361.

KEMPER, S. (1987). Syntactic complexity and elderly adults' prose recall. Experimental Aging Research, 13, 47-52.

KuČERA, H., \& FRANCIS, W. N. (1967). Computational analysis of present-day American English. Providence, RI: Brown University Press.

Light, L. L., \& ANDERSON, P. A. (1985). Working-memory capacity, age, and memory for discourse. Journal of Gerontology, 40, 737-747.

McDowD, J. M., \& CraIK, F. I. M. (in press). The effects of aging and task difficulty on divided attention performance. Journal of $E x$ perimental Psychology: Human Perception \& Performance.

Norman, D. A., Bobrow, D. G. (1975). On data-limited and resource-limited processes. Cognitive Psychology, 7, 44-64.

Salthouse, T. A., Rogan, J. D., \& Prill, K. (1984). Division of attention: Age differences on a visually presented memory task. Memory \& Cognition, 12, 613-620.

SPILICH, G. J. (1983). Life-span components of text processing: Structural and procedural differences. Journal of Verbal Learning \& Verbal Behavior, 22, 231-244.

Wason, P. C., Johnson-Laird, P. N. (1972). Psychology of reasoning: Structure and content. London: Batsford.

WELFORD, A. T. (1958). Aging and human skill. London: Oxford University Press.

W RIGHT, R. (1981). Aging, divided attention and processing capacity. Journal of Gerontology, 36, 605-614.

(Manuscript received April 15, 1987; revision accepted for publication December 21,1987 .) 